\title{
Pediatric COVID-19
}

\author{
Najim A. Jabir Al Awwadi* and Makarim M. Ali Hassan \\ Department of Pharmacology, Faculty of Pharmacy, Ministry of Higher Education, University of Thi-Qar, Dhi Qar, Iraq
}

${ }^{*}$ Corresponding author: Najim A. Jabir Al Awwadi, Department of Pharmacology, Faculty of Pharmacy, Ministry of Higher Education, University of Thi-Qar, Dhi Qar, Iraq; Tel: 07829300022; Email: dr.najimabbas@yahoo.fr

Received: April 28, 2021; Accepted: May 05, 2021; Published: May 13, 2021

\section{Virus Description}

Corona is a common viral disease that can be transmitted between humans and different types of animals, meaning it can be transmitted between different races and types of living organisms. It is characterized as having broad-spectrum disease symptoms that differ from one patient to another in their severity and type. In the last months of 2019, a storm of infection with the Corona virus appeared in the Chinese city of Wuhan, with symptoms that were almost different in severity and led to deaths in some infections. The virus was characterized by its rapid spread among people, which surprised researchers, doctors and people in that city and in China in general. Which challenged Chinese researchers and scientists to investigate the type and nature of the causative agent, so they were able to diagnose Corona virus (Cove 2).

Keywords: SARS cov2, Coronavirus, Children, COVID-19, Viral infection, Respiratory signs, Pfizer/BioNTech, Moderna and Johnson \& Johnson vaccines

\section{COVID 19 Susceptible Age of Children}

In December 2019, a novel coronavirus (severe acute respiratory syndrome coronavirus 2 [SARS-CoV-2]) emerged in China and has spread globally, creating a pandemic. Information about the clinical characteristics of infected patients who require intensive care is limited. The 2019 novel coronavirus (SARS-CoV-2) has been responsible for more than 54000000 infections and 1,200 000 deaths worldwide, but data regarding the epidemiologic characteristics and clinical features of infected children are limited [1,2]. The largest study so far, published in pediatrics J. included analysis of 2,143 children with COVID-19 documented from Jan. 16 to Feb. 8 in China. It found that symptoms of the disease were generally less severe in children and teens compared with adults. Specifically, 4.4 percent had no symptoms, 50.9 percent had mild disease and 38.8 percent had moderate symptoms. Of the children with symptoms, only 0.6 percent developed acute respiratory distress syndrome or multiple organ dysfunction. Of note, however, young children-particularly infants under one year of age-had a higher risk for significant illness. Ten percent of infants had severe disease, compared with 3 percent of teens over age 15 .

\section{How Likely are Children to Get Coronavirus Disease 2019 (COVID-19)?}

Although all children can be infected with the virus that causes COVID-19, they are not as frequent as adults. Children rarely encounter serious illnesses from COVID-19. Although there have been many large- scale outbreaks around the world, few children have died. According to the US Centers for Disease Control and Prevention (CDC), between February 12 and April 2, of the nearly 150,000 COVID-19 cases in the United States, only 2500, or $1.7 \%$, were children. This is similar to outbreaks in other countries such as China and Italy. The hospitalization rate of children is much lower than that of adults [3]. However, people of any age with certain underlying diseases (such as type 2 diabetes) have a higher risk of serious illnesses from COVID-19. In addition, children with congenital heart disease, genetic diseases, or diseases that affect the nervous system or metabolism are also at higher risk of serious illnesses from COVID-19. Discuss with them what happened and assure them that most situations are mild. Your child will get tips from you, so it's also important to stay calm.

\section{How Does COVID-19 have an Effect on Kids?}

Children, together with very younger kids, can infected with COVID-19. Many of them don't have any signs and symptoms. But those who do get the infection generally tend to show milder signs and symptoms which includes low to mild fever, exhaustion, and cough. Some kids have had marked fitness situations can be at expanded hazard for intense illness. A doubtlessly acute and perilous sequel can appear on kids. This case defined as multisystem inflammatory syndrome in children (MIS-C), it may result in life-threatening issues with the coronary heart and different organs with inside the frame. In this condition, exceptional frame parts, which includes the coronary heart, lungs, kidneys, brain, skin, eyes, or gastrointestinal organs, can end up inflamed.

\section{Symptoms of MIS-C can Include}

- Fever lasting more than a couple of days

- Rash

- Bloodshot eyes (redness of the white part of the eye)

- Stomach ache

- Vomiting and/or diarrhea

- A large, swollen lymph node in the neck

- Neck pain

- Red, cracked lips

- A tongue that is redder than usual and looks like a strawberry

- Swollen hands and/or feet

- Irritability and/or unusual sleepiness or weakness. 


\section{Why do Children React Differently to COVID-19?}

The answer is unclear. Some experts suggest that children may not be severely affected by COVID-19 because there are other coronaviruses that spread in the community and cause illness, such as the common cold. Since children often catch colds, they may have antibodies to protect them against COVID-19. Children's immune systems may also interact differently with adults' immune systems. Some adults get sick because their immune system seems to overreact to the virus, causing more damage to their bodies. This may be unlikely to occur in children. Although rare, children under 1 year old (infants) have a higher risk of serious illness from COVID-19. This may be due to their immature immune system and small respiratory tract, which makes them more susceptible to respiratory problems caused by respiratory viral infections. Between late December and early February, more than 2,100 children with suspected or confirmed COVID-19 in China were studied, and the results showed that less than $11 \%$ of infants had serious or severe illnesses. In contrast, the prevalence of severe or severe illness is about $7 \%$ for children aged 1 to 5 years, $4 \%$ for children 6 to 10 years old, $4 \%$ for children 11 to 15 years old, and $3 \%$ for children 16 years and older. New born babies may be infected with the virus that causes COVID-19 when they come into contact with sick caregivers during or after delivery. The American Academy of Pediatrics recommends special care for new-borns born to women who have confirmed or suspected COVID-19. This may include temporarily separating the mother from the new born to reduce the risk of infecting the baby, monitoring the baby for signs of infection, and, if available, testing the new born for COVID-19 [4,5].

\section{Do Children and Adults have Different Symptoms of COVID-19?}

When you see some mild symptoms on your son or daughter and feel or suspect that these symptoms are similar to those of COVID 19, you should take quick steps to isolate your child in a special room where all the comforts and conditions of health are available and prevent contact with him from the rest of the family and tell his or her doctor or health care providers and following the procedures recommended by the World Health Organization. COVID-19 symptoms in children and adults experience similar symptoms of COVID-19, while children's symptoms tend to be mild and cold. Most children will recover within one to two weeks. Their symptoms may include: fever Runny nose cough fatigue Muscle pain Vomiting diarrhoea. When children and adolescents get COVID-19, their symptoms seem to be milder than adults. Among the American population under 19, almost no one is hospitalized. Studies have shown that more than $90 \%$ of sick children have mild to moderate cold-like symptoms, including: fever Runny nose cough Vomiting diarrhoea. Some children and adolescents have been admitted to the hospital due to childhood multiple system inflammatory syndrome (MIS-C) or pediatric multiple system inflammatory syndrome (PMIS).

\section{Coronavirus in Sick Children if Some Children have Other Diseases}

They may be at higher risk of more serious diseases: asthma diabetes Blood disease Heart or liver disease Kidney disease requiring dialysis Weakened immune system. Doctors are still learning about it, but they think it is related to the new coronavirus. Symptoms include fever, abdominal pain, vomiting, diarrhea, skin rash, headache, and confusion. They are similar to toxic shock syndrome or Kawasaki disease, which causes inflammation of blood vessels in children. Serious problems are rare. If your child has any of the following symptoms, seek medical help immediately. Difficulty breathing Can't let the liquid flow down Changes in skin tone, including blue lips or face Confused or trouble waking up Serious problems are rare. If your child has any of the following symptoms, seek medical help immediately: Difficulty breathing Can't let the liquid flow down Confused or trouble waking up Blue lips or face.

\section{When Will Youngsters be Capable of Get the COVID-19 Vaccine?}

Pfizer/BioNTech and Moderna are already carrying out age deescalation researches, wherein the vaccines are examined in different categories of children of descending age. Johnson \& Johnson plans to do the same. Currently, the Pfizer/BioNTech COVID-19 vaccine is permitted to be used in teenagers sixteen years and older, but the Moderna and Johnson \& Johnson vaccines are given a permission for the young in 18 years and older. In March 2021, Pfizer/BioNTech introduced promising effects for a Phase three trial trying out its vaccine in youngsters a while 12 to 15 . Experiments were conducted on 2,260 voluntaries teenagers, half of whom were given mRNA vaccine and the rest were given Normal Saline or Placebo. The antibody reaction within side the vaccinated adolescent categories turned into even more potent than that during vaccinated sixteen- to 25 -year-olds enrolled in an in advance study. In addition, a complete of 18 symptomatic instances of COVID-19 have been said at some point of the trial, all within the placebo group. Vaccine-associated signs and symptoms have been slight and akin to older corporations enrolled in in advance study. The effects have been introduced in a press release, now no longer in a peer-reviewed, posted study. Pfizer/ BioNTech has submitted their facts to the FDA with a request to make bigger emergency use authorization to youngsters a while 12 to 15 . The enterprise has additionally commenced trying out the vaccine in children more youthful than 12 years. Moderna is carrying out vaccines study - one in adolescents a while 12 to 17 , the alternative in children among a while of 6 months and 12 years. The age de-escalation research is achieved to verify that the vaccines are secure and powerful for every age category. They additionally pick out the most reliable dose, which ought to be powerful, however with tolerable facet effects. The adult trail is a greater than the age de-escalation study in the children; in addition of recruiting many thousands of contributors, they may recruit between 2,000-3000 participants in every age category. Look like the adult studies, a few children in every study gets a placebo. The FDA will evaluation facts from the de-escalation trials to determine whether or not to authorize the vaccines for every age category.

\section{References}

1. World Health Organization. Coronavirus disease 2019 (COVID-19): situation report -50 .

2. Guan W, Ni Z, Hu Y, Wen-hua Liang, Chun-quan OU, et al. (2020) Clinical characteristics of coronavirus disease 2019 in China. N Engl J Med 382: 1708-1720. 
3. Bialek S, Boundy E, Bowen V, et al. (2020) CDC COVID-19 Response Team. Severe outcomes among patients with coronavirus disease 2019 (COVID-19)-United States, February 12-March 16, 2020. MMWR Morb Mortal Wkly Rep 69: 343-346.

4. Ng Y, Li Z, Chua YX, et al. (2020) Evaluation of the effectiveness of surveillance and containment measures for the first 100 patients with COVID-19 in SingaporeJanuary 2-February 29, 2020. MMWR Morb Mortal Wkly Rep 69: 307-311.
5. The Novel Coronavirus Emergency Response Epidemiology Team (2020) The epidemiological characteristics of an outbreak of 2019 novel coronavirus diseases (COVID-19)-China. China CDC Weekly 2: 113-122.

\section{Citation:}

Jabir Al Awwadi NA, Ali Hassan MM (2021) Pediatric COVID-19. J Pharmacol Pharm Res Volume 4(2): 1-3. 